University of Nebraska - Lincoln

DigitalCommons@University of Nebraska - Lincoln

\title{
Magnetism of Fe clusters formed by buffer-layer assisted growth on Pt(997)
}

\section{J. Zhang}

Max-Planck-Institut f"ur Festk"orperforschung, Heisenbergstrasse 1, 70569 Stuttgart, Germany

D. Repetto

Max-Planck-Institut f"ur Festk"orperforschung, Heisenbergstrasse 1, 70569 Stuttgart, Germany

V. Sessi

Max-Planck-Institut f"ur Festk"orperforschung, Heisenbergstrasse 1, 70569 Stuttgart, Germany, violetta.sessi@jku.at

Jan Honolka

Max-Planck-Institut für Festkörperforschung, honolka@fzu.cz

Axel Enders

University of Nebraska at Lincoln, a.enders@me.com

See next page for additional authors

Follow this and additional works at: https://digitalcommons.unl.edu/physicsenders

Part of the Physics Commons

Zhang, J.; Repetto, D.; Sessi, V.; Honolka, Jan; Enders, Axel; and Kern, Klaus, "Magnetism of Fe clusters formed by buffer-layer assisted growth on Pt(997)" (2007). Axel Enders Publications. 16.

https://digitalcommons.unl.edu/physicsenders/16

This Article is brought to you for free and open access by the Research Papers in Physics and Astronomy at DigitalCommons@University of Nebraska - Lincoln. It has been accepted for inclusion in Axel Enders Publications by an authorized administrator of DigitalCommons@University of Nebraska - Lincoln. 


\section{Authors}

J. Zhang, D. Repetto, V. Sessi, Jan Honolka, Axel Enders, and Klaus Kern

This article is available at DigitalCommons@University of Nebraska - Lincoln: https://digitalcommons.unl.edu/ 


\title{
Magnetism of Fe clusters formed by buffer-layer assisted growth on $\operatorname{Pt}(997)$
}

\author{
J. Zhang ${ }^{1}$, D. Repetto ${ }^{1,2}$, V. Sessi ${ }^{1}$, J. Honolka ${ }^{1}$, A. Enders ${ }^{1, a}$, and K. Kern ${ }^{1}$ \\ 1 Max-Planck-Institut für Festkörperforschung, Heisenbergstrasse 1, 70569 Stuttgart, Germany \\ 2 Universidad de Valencia, Instituto de Ciencia Molecular, Polígon de la Coma s/n, 46980 Paterna, Spain
}

Received 31 January 2007 / Received in final form 26 April 2007

Published online 13 June 2007 - (c) EDP Sciences, Società Italiana di Fisica, Springer-Verlag 2007

\begin{abstract}
The growth and magnetism of nanometer size Fe clusters on stepped Pt surfaces is investigated by scanning tunneling microscopy (STM) and magneto-optical Kerr effect measurements (MOKE). The clusters are formed on xenon buffer layers of varying thickness and then brought into contact with the substrate by thermal desorption of the Xe. The cluster size is controlled by the thickness of the Xe layer. It is found that clusters of diameter smaller than the Pt terrace width of $2 \mathrm{~nm}$ are aligned along the step edges of the $\mathrm{Pt}(997)$, thus forming linear cluster chains. In this arrangement, the clusters are ferromagnetic with an easy axis in the direction along the surface normal. If the cluster diameter is larger than the terrace width then the alignment along the step edges is not observed and rather large agglomerates are found which are randomly distributed over the surface. Despite their increased volume, such agglomerates are superparamagnetic with in-plane easy magnetization axis. The enhanced magnetic anisotropy energy in the smallest clusters is originating from hybridization effects at the Fe-Pt interface.
\end{abstract}

PACS. 61.46.-w Nanoscale materials - 68.37.Ef Scanning tunneling microscopy - 36.40.Cg Electronic and magnetic properties of clusters - 75.75.+a Magnetic properties of nanostructures

\section{Introduction}

Metal clusters and small nanoparticles currently attract particular interest in nano-scale research, as they can be prepared easily and of virtually any size in the nanometer size range. Within this range, fundamental physical properties, such as magnetism, the electronic structure, optical properties or the chemical reactivity, change from truly atomistic behavior to bulk properties $[1,2]$. Concerning magnetism, characteristic length scales range from $1 \AA$ (exchange) over a few nanometers (RKKY interaction) up to several $\mu \mathrm{m}$ (domains in hard and soft ferromagnets). The systematic study of clusters contributes therefore to a fundamental understanding of magnetism as they allow to follow its evolution when crossing size ranges in which different interactions are predominant.

The properties of clusters in contact with surfaces are not only determined by pristine size effects but they are in addition substantially affected by the interaction with the substrate [3-5]. Surface free energies, lattice mismatch, diffusion barriers or structural imperfections can determine the crystalline structure and morphology of the clusters. In addition, hybridization with the substrate alters the electronic structure [6-8]. The substrate can thus be used to influence the physical or chemical properties of clusters.

\footnotetext{
a e-mail: a.enders@fkf .mpg.de
}

Two techniques have been developed to deposit compact clusters on single crystalline substrates under ultrahigh vacuum conditions, which are the soft-landing of clusters formed in the gas phase [9] and the noble gas buffer layer assisted cluster growth (BLAG) [10-12]. Both techniques are, in principle, suitable to form clusters of almost any material on any substrate $[13,14]$. The clusters are formed before they make contact with the substrate and their initial structure is not impeded by the surface [8]. However, the final shape and structure as well as the size and spatial distribution of the clusters after landing can be strongly affected by surface wetting and diffusivity. The main advantage of BLAG over other cluster deposition methods is that no experimental equipment beyond the standard molecular beam epitaxy (MBE) tools are required. Furthermore, the formation of the cluster layer is a parallel process and high cluster coverage can be achieved during a short preparation step. In contrast, the cluster flux of dedicated cluster sources is limited by the mass filtering. This increases the deposition time considerably, but gives usually narrower cluster size distribution. Recent overviews over fabrication and properties of surface supported clusters can be found, for instance, in references $[3,15]$.

In this paper we present a comprehensive study of dispersed Fe clusters produced by buffer-layer assisted growth on stepped Pt surfaces. Morphology and magnetism of the clusters are systematically monitored as a 
function of the buffer layer thickness. We show that the clusters attach to the substrate step edges and form linear cluster chains if their diameter matches the distance between neighboring steps on the $\operatorname{Pt}(997)$ surface. For such small clusters the substrate contributions to the magnetic anisotropy are found to govern the overall magnetic behavior. The present work offers therefore the prospect of fabricating well-defined, ordered cluster arrangements with controlled magnetic properties by using template surfaces.

\section{Experimental}

The experiments were performed in an ultrahigh vacuum (UHV) chamber which combines in-situ scanning tunneling microscopy (STM) and magneto-optical Kerr effect (MOKE) experiments, as well as experiments for lowenergy electron diffraction (LEED) and Auger electron spectroscopy (AES) [16]. The sample temperature could be adjusted between $35 \mathrm{~K}$ and $900 \mathrm{~K}$ for the preparation and between $35 \mathrm{~K}$ and $300 \mathrm{~K}$ for the MOKE measurements. All STM experiments shown in this paper are done at $300 \mathrm{~K}$.

$\mathrm{Pt}(997)$ single crystals were used as substrates for the present work. Pt(997) can be easily prepared under ultrahigh vacuum conditions and has been demonstrated to be useful as nanotemplate for supported growth $[17,18]$. The substrate surface is composed of (111) oriented terraces of $20.2 \AA$ in width, which are separated by mono-atomic steps [19]. The regular step ordering is mediated by repulsive step-step interactions. The substrates were prepared by repeated cycles of Ar ion sputtering and annealing to $870 \mathrm{~K}$. The preparation was finished when sharp superstructure diffraction spots from the regular step-terrace ordering on the $\mathrm{Pt}(997)$ surface were observed by LEED over the entire surface area. The substrate and adlayer cleanliness as well as the adlayer coverage was monitored by AES [20].

For the fabrication of Fe clusters we followed a procedure that is described in detail elsewhere [8]. In short, Fe deposited on the substrate is forming small clusters if the substrate has been pre-covered with a xenon noble gas layer at 35-40 K [21]. Thermal desorption of the Xe at $T=100 \mathrm{~K}$ brings the clusters into contact with the substrate. The final cluster size depends on the Xe thickness and on the Fe coverage.

The thickness of the Xe buffer layer is controlled by adjusting the Xe partial pressure and the exposure time. It has been demonstrated that a Xe gas flow of 5.5 Langmuir $\left(1 \mathrm{~L}=10^{-6}\right.$ Torr $\left.\times 1 \mathrm{~s}\right)$ leads to the formation of $1 \mathrm{ML}$ Xe, assuming a sticking coefficient of 1 at the deposition temperature of $35 \mathrm{~K}$ [12]. However, the sticking coefficient might become smaller with increasing Xe thickness due to limited heat transfer through the noble gas layer. Reliable measurements of the Xe coverage as a function of Xe exposure for large coverage are not available. We therefore quote the Xe coverage, $\theta_{\mathrm{Xe}}$, in units of Langmuir (L) throughout the paper.

Fe was evaporated from a rod with a purity of $99.99 \%$ using an electron beam evaporator. The Fe deposition rate
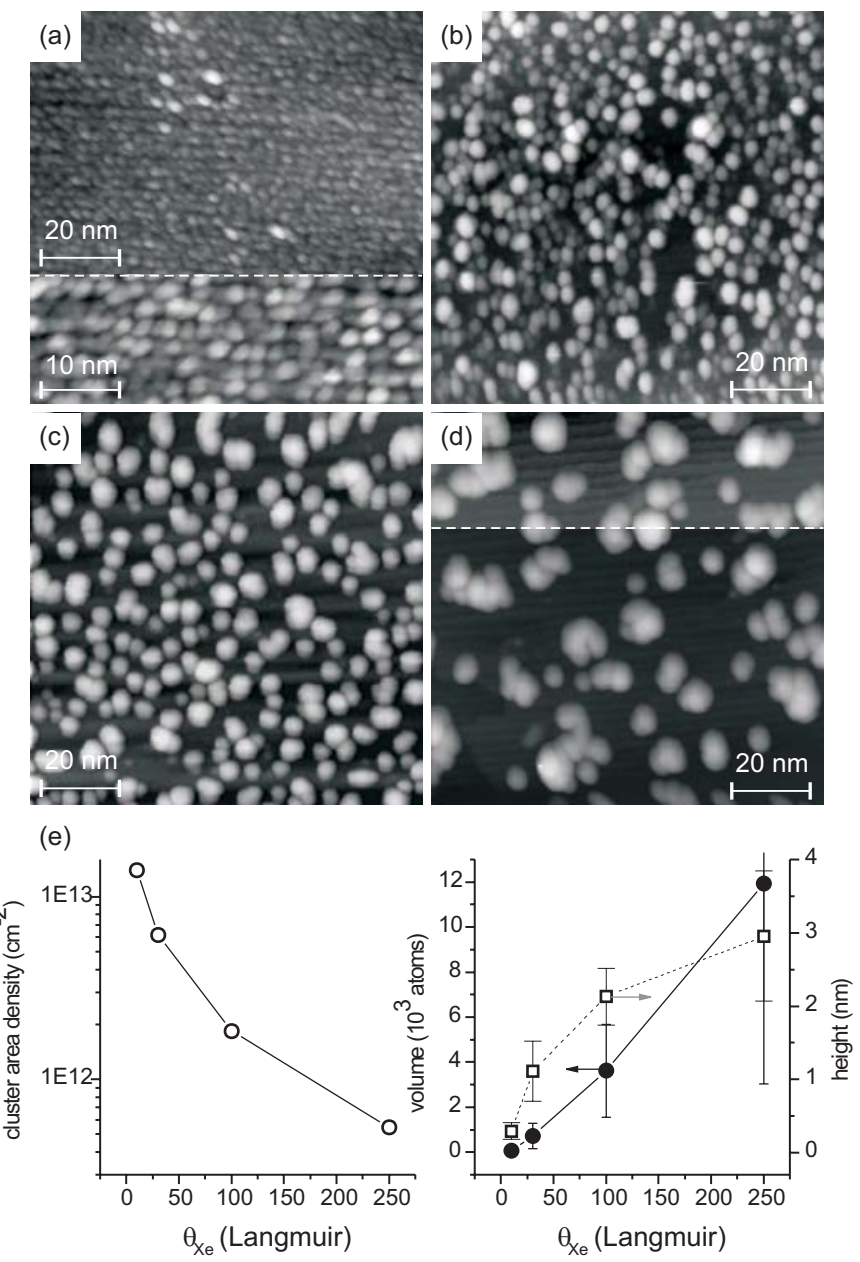

Fig. 1. STM images of Fe clusters on $\mathrm{Pt}(997)$, prepared with $2 \mathrm{ML} F \mathrm{Fe}$ and different Xe buffer layer thickness. (a) $\theta_{\mathrm{Xe}}=10 \mathrm{~L}$, the bottom part shows a region scanned with higher magnification $(\times 2)$, (b) $\theta_{\mathrm{Xe}}=30 \mathrm{~L}$, (c) $\theta_{\mathrm{Xe}}=100 \mathrm{~L}$, (d) $\theta_{\mathrm{Xe}}=250 \mathrm{~L}$, the contrast in the top part of this image has been adjusted to enhance the visibility of the substrate steps, (e) dependence of cluster area density, cluster height $(\square)$ and volume $(\bullet)$ on $\theta_{\mathrm{Xe}}$, with $\theta_{\mathrm{Fe}}=2 \mathrm{ML}$. The $y$-bars in the plot to the right represent the FWHM values of the volume and height distributions. The number of atoms per clusters has been calculated from the measured cluster volume by using bulk Fe density, 84.6 atoms per $\mathrm{nm}^{3}$, without correcting for tip convolution effects.

was calibrated by a quartz microbalance prior to each deposition. A nominal Fe coverage of $\theta_{\mathrm{Fe}}=1 \mathrm{ML}$ denominates an atomic density of $1.21 \times 10^{15}$ atoms $/ \mathrm{cm}^{2}$, which corresponds to the atomic density of a relaxed bcc Fe(100) layer of 1.43 Angstrom thickness.

\section{Results}

We investigated Fe clusters formed of a constant Fe coverage, $\theta_{\mathrm{Fe}}=2 \mathrm{ML}$, as a function of the Xe coverage, $\theta_{\mathrm{Xe}}$. STM images of clusters formed with $10 \mathrm{~L}(1.8 \mathrm{ML}), 30 \mathrm{~L}$ (5.5 ML), $100 \mathrm{~L}(18 \mathrm{ML})$, and $250 \mathrm{~L}$ (45 ML) Xe are shown in Figures 1a-1d. Evidently, the higher $\theta_{\mathrm{Xe}}$, the larger the 


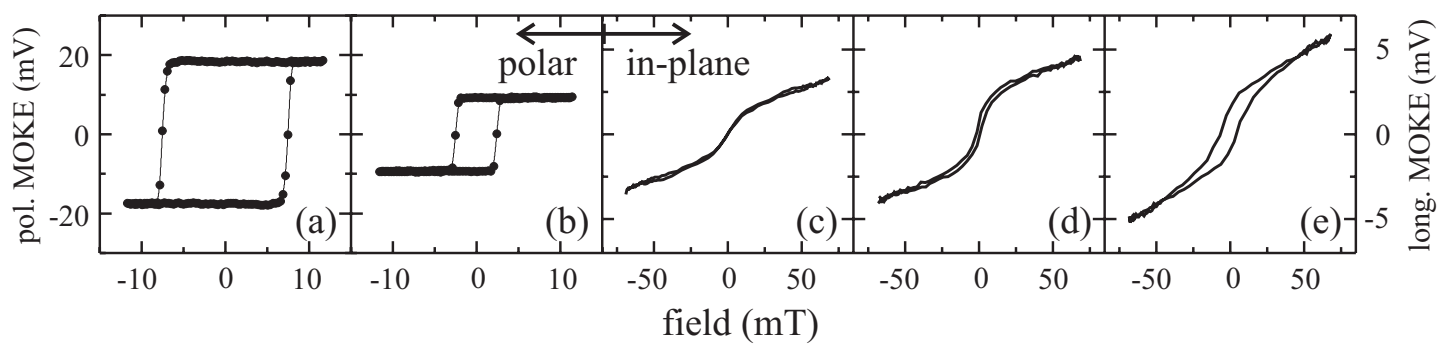

Fig. 2. Magneto-optical Kerr effect measurements (MOKE) on a 2 ML Fe film epitaxially grown on Pt(997) (a); clusters of 2 ML Fe prepared with 10 L Xe (b), 30 L Xe (c), 100 L Xe (d), 250 L Xe (e). The loops in (a, b) have been measured with polar MOKE (out-of-plane), the loops in (c-e) with longitudinal MOKE (in-plane). The substrate temperature was $40 \mathrm{~K}$ for all samples during Fe deposition, and $147 \mathrm{~K}(\mathrm{a}), 115 \mathrm{~K}(\mathrm{~b})$ and $47 \mathrm{~K}(\mathrm{c}-\mathrm{e})$ during MOKE.

clusters. The arrangement of the clusters depends on their size. For the lowest $\theta_{\mathrm{Xe}}=10 \mathrm{~L}$ the clusters appear of $\approx 2 \mathrm{~nm}$ diameter at the base and $2-3$ atomic Fe layers in height after landing on the Pt substrate. Their diameter corresponds to the width of the substrate terraces, therefore each terrace can accommodate only one cluster in the direction perpendicular to the step edges. As a result, the clusters are aligned into linear chains. The density of the clusters is 14.5 clusters per $10 \times 10 \mathrm{~nm}^{2}$ area and neighboring clusters seem to be in contact with each other at their bases.

Already with $30 \mathrm{~L}$ Xe the cluster size is larger than the terrace width $(\varnothing=3.9 \pm 2.8 \mathrm{~nm})$ and hence the terrace cannot promote chain-like ordering of the clusters as in (a). Isolated clusters of diameter $\varnothing=6.7 \pm 4.2 \mathrm{~nm}$ are formed with $\theta_{\mathrm{Xe}}=100 \mathrm{~L}$. For $\theta_{\mathrm{Xe}}=250 \mathrm{~L}$ the cluster size is further increased to $\varnothing=9.9 \pm 7.6 \mathrm{~nm}$, and typically cluster agglomerates are found. The individual clusters constituting the large agglomerates in $(\mathrm{d})$ remain still visible. It appears as if the clusters which are in direct contact with each other are unable to merge into one aggregate, as it would be expected at room temperature from a thermodynamic point of view.

The magnetism of the clusters has been studied with longitudinal and polar magneto-optical Kerr effect measurements (MOKE). Hysteresis loops of samples equivalent to those in Figure 1 are shown in Figure 2. While magnetization loops have been taken for each sample along out-of-plane and in-plane directions we show only those loops that we identified as easy axis loops from the loop shape. This is the out-of-plane direction for the samples in Figures 2a, 2b and the in-plane direction for the samples in (c-e). Two monolayers of Fe deposited on $\mathrm{Pt}(997)$ without Xe buffer layer show square shaped magnetization loops with pronounced perpendicular easy axis (a), as a result of the dominant Fe-Pt interface contribution to the total anisotropy energy [20]. The temperature of this sample was somewhat increased for the MOKE measurements. The coercivity was thus sufficiently reduced so that the magnetization could be switched with the available maximum field.

With increasing cluster size the magnetization loops evolve gradually from the square-shape towards a pronounced S-shape. For the linear cluster chains formed with only $10 \mathrm{~L}$ of Xe the easy axis is still out-of-plane and square shaped, albeit with reduced total Kerr signal in saturation (b). As soon as the registry of the clusters with the substrate steps is lost the overall magnetic behavior corresponds to that of a superparamagnetic ensemble. Here, the maximum available field of $70 \mathrm{mT}$ is not sufficient to magnetically saturate the sample at $47 \mathrm{~K}$ due to thermal fluctuations of the macrospins, as well as a distribution of the cluster size and orientation. At this temperature, blocking is observed in the form of open magnetization loops with remanent magnetization at zero field only for the largest clusters in (e). However, the increase of the mean cluster size with Xe buffer layer thickness is reflected in the increase of the measured magnetization value at a given field and temperature.

The blocking temperature, $T_{B}$, can be determined from the temperature dependence of the magnetization loops. In Figure 3 (top) the Kerr signal at maximum field $(\circ)$ and zero field $(\bullet)$ as well as the coercivity $(+)$ are plotted for clusters formed with $\theta_{\mathrm{Fe}}=2 \mathrm{ML}$ and $\theta_{\mathrm{Xe}}=250 \mathrm{~L}$ (as those in Figs. 1d and 2e). As an approximation we identify $T_{B}$ as the temperature at which the remanence becomes zero. This is $80 \mathrm{~K}$ for the present sample. For comparison, the same experiment is shown for larger clusters formed of $\theta_{\mathrm{Fe}}=4 \mathrm{ML}$ and $\theta_{\mathrm{Xe}}=100 \mathrm{~L}$ in Figure 3 (bottom). Here, the blocking temperature is found to be increased to $T_{B}=100 \mathrm{~K}$, as a result of the increased cluster volume. A more precise experimental determination of $T_{B}$ can, however, be obtained from temperature dependent susceptibility measurements [22].

\section{Discussion}

Important results of this study are (i) the determination of the $\theta_{\mathrm{Xe}}$ - dependence of the size of Fe clusters on Pt(997) substrates; (ii) the alignment of small clusters into chains along substrate steps; and (iii) the determination of the magnetic properties of supported Fe clusters as a function of their size. These results will be discussed in the following.

The alignment of the smallest clusters in this study along the step edges of the substrate is ascribed to the attractive potential there. Step edges are well-known to provide energetic sinks for atomic diffusion. The higher 


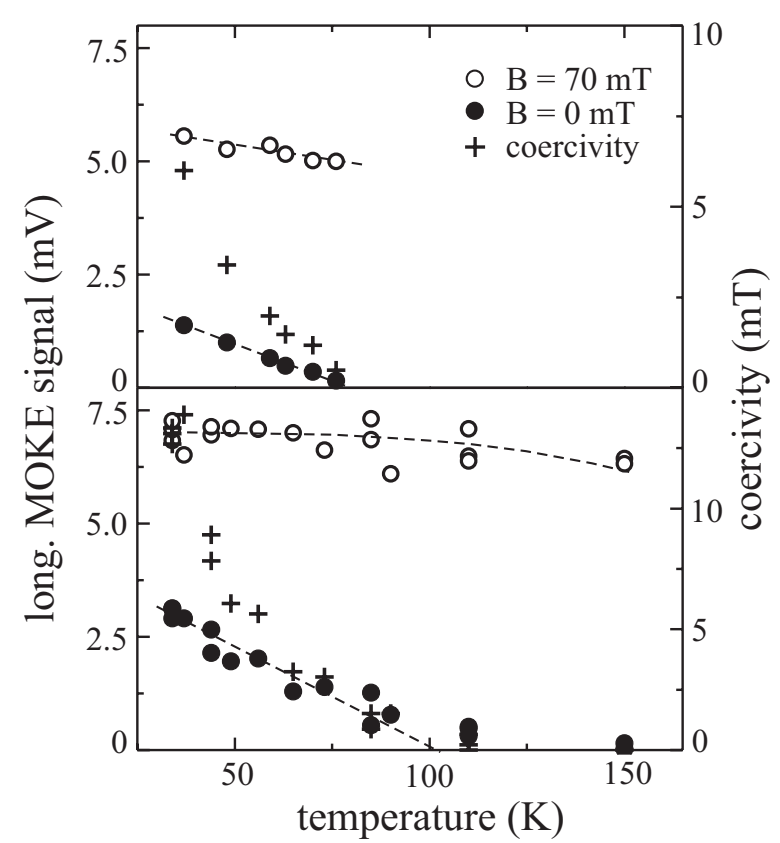

Fig. 3. Temperature dependence of the magnetization of Fe clusters on $\mathrm{Pt}$ (997) formed with $2 \mathrm{ML} \mathrm{Fe} / 250 \mathrm{~L}$ Xe (top panel) and $4 \mathrm{ML} \mathrm{Fe} / 100 \mathrm{~L} \mathrm{Xe} \mathrm{(bottom} \mathrm{panel)} \mathrm{(from} \mathrm{[8]).} \mathrm{The} \mathrm{data} \mathrm{for}$ remanence $(\bullet), \mathrm{M}$ at $70 \mathrm{mT}(\mathrm{\circ})$ and coercivity $(+)$ have been taken from magnetization loops measured with longitudinal MOKE.

binding energy at the steps and the diffusivity of atoms along the steps ensures discrete row-by-row growth during MBE growth [23,24]. Stepped surfaces have therefore been exploited as templates to grow quasi-one-dimensional wires of atoms $[17,18,20]$. We have experimental evidence that not only atoms, but also small clusters with diameters of $\approx 1-2 \mathrm{~nm}$ are mobile on various metal surfaces at $300 \mathrm{~K}$ after Xe desorption. Those clusters would have the possibility to reach the step edges, where their mobility is reduced. In this picture, linear chains of clusters are formed if the cluster's diameter matches the terrace width. One might speculate if the step decoration occurs already on the Xe buffer layer. A $10 \mathrm{~L}$ Xe exposure results in a Xe film of only 2 monolayer thickness. Such a thin $\mathrm{Xe}$ film is expected to mimic the steps of the substrate, thus representing lines of defects equivalent to the substrate steps. Atomic motion and cluster formation might therefore be influenced already on the buffer layer by the Xe steps. Although the exact role of the steps remains to be investigated the experiments demonstrate that a template effect can, in principle, be achieved with stepped crystalline surfaces also for small clusters. This templating fails when thicker Xe layers are used and the clusters become larger than the terrace width. This is already the case for a Xe coverage of $30 \mathrm{~L}(\approx 5 \mathrm{ML})$.

A demonstrative picture of the cluster formation process is given in references $[11,12]$. It has been argued that the different surface energies of the metal and Xe inhibit monolayer formation and promote the formation of very small clusters. The clusters become highly mobile during
Xe desorption and merge into larger clusters at the same time. The thicker the Xe layer, the larger the resulting clusters, and the lower their area density $[25,26]$. We observe roughly a linear dependence of the cluster volume on the Xe coverage (Fig. 1e). However, the cluster area densities found in this study are one order of magnitude larger than the densities of other metal clusters found in reference [26] for similar Xe and metal coverages. Also, no ramified cluster shapes are found for Fe in this study, but instead rather large agglomerates of clusters as in Figure 1d.

The perpendicular magnetization axis found for $\mathrm{Fe}$ films and small clusters in Figures 2a, 2b is ascribed to the electronic hybridization at the Fe-Pt interface. The magnetic anisotropy of the Fe atoms in direct contact with $\mathrm{Pt}$ is enhanced (see paper by Bornemann et al. in this issue). This interface anisotropy is sufficiently large to govern the magnetization of small clusters of only $0.1 \mathrm{ML}$ Fe on $\mathrm{Pt}(111)$ [27] and of epitaxial Fe films up to a thickness of 3.3 monolayer on $\mathrm{Pt}(997)$ [20]. The mean cluster height in Figure 1a of 2.1 ML (3.0 $\AA$ ) is below the critical thickness for spin reorientation, hence the out of plane easy axis. The Fe-Pt interface seems to increase the total anisotropy energy sufficiently to block the cluster spins against thermal fluctuation. Already for the clusters in Figure $1 \mathrm{~b}$ the average height is with $\approx 8 \mathrm{ML}$ well above the critical thickness, and in-plane easy axis as well as superparamagnetic behavior is found, accordingly.

It is interesting to note that the change from ferromagnetism to superparamagnetism coincides with the loss of the registry of the clusters with the substrate steps. It is likely that some of the clusters in the one-dimensional chains are in direct contact at their bases. In this case the individual macrospins are exchange coupled, further contributing to the ferromagnetic loop shape. The exchange interaction would dominate by far the magnetic behavior, even though the total magnetic anisotropy energy (MAE) is expected to be largest for the smallest clusters due to the interface contribution. For the larger separated clusters in Figures 1c, 1d this exchange interaction between clusters is not possible. Here the MAE is determined by the competition between interface, bulk and shape anisotropy contributions, which results in in-plane magnetization.

In superparamagnetic ensembles the individual magnetic moments of the clusters become decoupled at the blocking temperature due to thermal fluctuations. The blocking temperature of non-interacting clusters depends on the MAE and the magnetic volume, $V$, and can be used to estimate the cluster size according to $V \approx 25 k_{B} T_{B} E_{A}^{-1}$ $\left(k_{B}\right.$ : Boltzmann constant) [28]. By inserting Fe bulk MAE values for the energy barrier $E_{A}$ and assuming spherical cluster shape we obtain mean cluster diameters of $9.7 \mathrm{~nm}$ $\left(4.0 \times 10^{4}\right.$ atoms $)$ and $10.4 \mathrm{~nm}\left(5.0 \times 10^{4} \mathrm{Fe}\right.$ atoms $)$ from the temperature dependent MOKE measurements on nominally deposited $2 \mathrm{ML}$ and $4 \mathrm{ML} \mathrm{Fe}$, as shown in Figure 3. An overview over the results from other samples with different $\theta_{\mathrm{Fe}, \mathrm{Xe}}$ is given in Table 1 .

We can use the STM study in Figure 1 to scrutinize the cluster diameter calculated from $T_{B}$. For the Fe 
Table 1. Average diameters of spherical Fe clusters as a function of $\theta_{\mathrm{Fe}}$ and $\theta_{\mathrm{Xe}}$. Values are calculated from the measured blocking temperature assuming Fe bulk MAE. The given numbers of atoms per cluster, $N$, are based on bulk Fe density and bcc crystalline structure.

\begin{tabular}{lllll}
\hline$\theta_{\mathrm{Fe}}(\mathrm{ML})$ & $\theta_{\mathrm{Xe}}(\mathrm{L})$ & $T_{B}(\mathrm{~K})$ & $\varnothing(\mathrm{nm})$ & $N$ \\
\hline 2 & 100 & 50 & 8.3 & $2.4 \times 10^{4}$ \\
2 & 250 & 80 & 9.7 & $4.0 \times 10^{4}$ \\
4 & 30 & 55 & 8.6 & $2.8 \times 10^{4}$ \\
4 & 100 & 100 & 10.4 & $5.0 \times 10^{4}$ \\
\hline
\end{tabular}

clusters fabricated with $\theta_{\mathrm{Fe}}=2 \mathrm{ML}$ and $\theta_{\mathrm{Xe}}=250 \mathrm{~L}$ we find some disagreement between the diameter calculated from $T_{B}$ of $9.7 \mathrm{~nm}$ and the diameter obtained from STM measurements of $6.5 \mathrm{~nm}$. The latter diameter corresponds to a sphere which has the same volume as the flattened hemispheres seen in the STM image in Figure 1d. It is likely that the STM is even overstating the cluster volume due to tip convolution effects so that $6.5 \mathrm{~nm}$ is actually an upper limit for the mean diameter of the spherical clusters.

The disagreement between the cluster volume from the blocking temperature and the STM data on the cluster volume confirms now also for this system that the MAE of small clusters is enhanced with respect to bulk material. Recent experiments as well as Monte Carlo simulations have already demonstrated that the use of the bulk anisotropy values for clusters of this size may lead to an overestimation for the volume since the large surface to volume ratio can noticeably increase the total anisotropy energy $[6,29]$. Besides such a size effect, the net magnetization of a cluster ensemble can also be stabilized by inter-particle and particle-substrate interactions $[6,7,30,31]$. Dipole-dipole interaction between $\mathrm{Fe}$ clusters of only 13 percent area coverage alone was found to raise the blocking temperature by $20 \mathrm{~K}[6]$.

We expect that in the present case, Fe clusters on $\mathrm{Pt}$, the dominant contribution to the MAE is coming from the interaction of the clusters with the substrate. The clusters hybridize with the underlying substrate, thus producing a cloud of magnetic polarization in the Pt $[32,33]$. Although the contact to the substrate somewhat reduces the average spin moment per atom in the cluster, it introduces a moment in the $d$-band of the substrate, which might even be extended beyond the region of physical contact [34]. Hence, the effective magnetic volume as well as the anisotropy are increased, which stabilizes the overall magnetization, increases the blocking temperature and ties the cluster magnetization to the substrate lattice.

The calculation of the cluster size from $T_{B}$ provides therefore in general only an estimate and may yield values which deviate from the actual volume by as much as one order of magnitude. We expect that the disagreement between cluster sizes measured by STM and calculated from $T_{B}$ becomes the larger the smaller the clusters are and the higher their area density.

\section{Conclusion}

The presented work shows that small Fe clusters formed with buffer layer assisted growth are arranged along the mono-atomic step edges of $\mathrm{Pt}(997)$ surfaces. We have therefore demonstrated that $\mathrm{Pt}(997)$ can be a useful template surface also for larger aggregates, besides its known template effect for single atoms. Our results suggest that also other cluster arrangements could be feasible by using more complex template surfaces, such as surface reconstructions, self-assembled structured surfaces or molecular layers. The $\mathrm{Pt}(997)$ surface determines also the magnetic properties of the clusters directly via cluster-substrate interaction, and indirectly by controlling the cluster size and distribution. Our work directs therefore the way towards ordered self-assembled structures with magnetic functionality.

We would like to thank the Deutsche Forschungsgemeinschaft for financial support within SPP 1153, "Cluster in contact with surfaces: electronic structure and magnetism".

\section{References}

1. T.P. Martin, Phys. Rep. 273, 199 (1996)

2. W.A. de Heer, Rev. Mod. Phys. 65, 611 (1993)

3. J. Bansmann et al., Surf. Sci. Rep. 56, 189 (2005)

4. P. Lang, R. Zeller, K. Wildberger, V.S. Stepanyuk, P.H. Dederichs, Phys. Rev. Lett. 75, 509 (1995)

5. S. Pick, G.M. Pastor, J. Dorantes-Dávila, H. Dreyssé, Phys. Rev. Lett. 75, 326 (1995)

6. D.P. Landau, G. Brown, J.P. Pierce, Z.Gai, G.A. Farnan, H.K. Lee, T.C. Schulthess, J. Shen, J. Appl. Phys. 91, 6926 (2002)

7. Z. Gai, J. Shi, T. Schulthess, G. Farnan, J. Wendelken, E. Plummer, J. Pierce, M. Torija, Phys. Rev. Lett. 92, 237201 (2004)

8. D. Repetto, J. Honolka, S. Rusponi, H. Brune, A. Enders, K. Kern, Appl. Phys. A 82, 109 (2006)

9. H. Brune, W. Harbich, R. Monot, J. Buttet, K. Kern, K. Bromann, C. Felix, Science 274, 956 (1996)

10. J.H. Weaver, G.D. Waddill, Science 251, 1444 (1991)

11. J.H. Weaver, L. Huang, S.J. Chey, Phys. Rev. Lett. 80, 4095 (1998)

12. J.H. Weaver, C. Haley, Surf. Sci. 518, 243 (2002)

13. J.H. Weaver, V.N. Antonov, Surf. Sci. 557, 1 (2004)

14. G. Kerner, M. Asscher, Surf. Sci. 557, 5 (2004)

15. E.W. Plummer, J. Shen, J.P. Pierce, J. Kirschner, J. Phys.: Condens. Matter 15, R1 (2003)

16. D. Peterka, A. Enders, G. Haas, K. Kern, Rev. Sci. Instrum. 74, 2744 (2003)

17. K. Maiti, M.C. Malagoli, W. Eberhardt, K. Kern, P. Gambardella, A. Dallmeyer, C. Carbone, Nature 416, 301 (2002)

18. K. Kuhnke, T.-Y. Lee, S. Sarbach, K. Kern, Surf. Sci. 600 , 3266 (2006)

19. V. Marsico, A. Fricke, E. Hahn, H. Schief, K. Kern, Phys. Rev. Lett. 72, 3378 (1994) 
20. S. Rusponi, J. Honolka, K. Kuhnke, V. Sessi, U. Starke, H. Brune, P. Gambardella, C. Carbone, A. Enders, D. Repetto, T.Y. Lee, K. Kern, Phys. Rev. B 74, 054408 (2006)

21. R. David, K. Kern, P. Zeppenfeld, G. Comsa, Surf. Sci. 195, 353 (1988)

22. N. Weiss, M. Epple, P. Buluschek, L. Claude, S. Rusponi, T. Cren, H. Brune, Nature Mat. 2, 546 (2003)

23. H. Brune, K. Kuhnke, P. Gambardella, M. Blanc, K. Kern, Phys. Rev. B 61, 2254 (2000)

24. K. Kuhnke, V. Marsico, M. Blanc, K. Kern, Phys. Rev. Lett. 78, 94 (1997)

25. A.S. Bhatti, V.N. Antonov, J.S. Palmer, J.H Weaver, Phys. Rev. B 68, 205418 (2003)

26. P.S. Waggoner, A.S. Bhatti, V.N. Antonov, J.S. Palmer, J.H. Weaver, Phys. Rev. B 70, 045406 (2004)

27. J. Honolka, A. Enders, K. Kern, V. Sessi, J. Zhang (unpublished)
28. Chikazumi, Physics of Ferromagnetism, 2nd edn. (Oxford University Press, 1997), p. 546

29. S.H. Baker, S.C. Thornton, C. Norris, J.B. Goedkoop, M. Finazzi, K.W. Edmonds, C. Binns, N.B. Brookes, Phys. Rev. B 60, 472 (1999)

30. D. Kechrakos, K. Trohidou, Phys. Rev. B 58, 12169 (1998)

31. H. Shima, Y. Otani, S.G. Kim, K. Fukamichi, N. Kikuchi, O. Kitakami, Y. Shimada, V. Novosad, K.Y. Guslienko, Phys. Rev. B 65, 60402 (2002)

32. K. Wildberger, R. Zeller, P.H. Dederichs, V.S. Stepanyuk, W. Hergert, Phys. Rev. B 53, 2121 (1996)

33. M. Komelj, C. Ederer, M. Fähnle, Phys. Rev. B 68, 52402 (2003)

34. R. López-Sandoval, R. Garibay-Alonso, Solid State Comm. 134, $503(2005)$ 